\title{
A Cross-Sectional Study of Prescribing Pattern of Antimicrobial Agents Among Inpatients of a Tertiary Healthcare Centre
}

\author{
Monica Jain, ${ }^{1}$ Shivankan Kakkar, ${ }^{1}$ Alka Bansal, ${ }^{1}$ Rupa Kapadia ${ }^{1}$
}

\begin{abstract}
Background: Antimicrobial agents are the mainstay of treatment in modern medicine. In view of emerging threat of Antimicrobial Resistance (AMR), there was a requirement aimed at identifying patterns of antimicrobial prescribing. The prescribing pattern of antimicrobial agents (AMAs) among the medicine inpatients of SMS Medical College \& Hospital, Jaipur, India was studied. The objectives were to evaluate, monitor and suggest rational prescribing practices.

Methods: This was a cross-sectional study that was done for a period of one year from July 2016 to June 2017. The rationality of antimicrobial drug use was evaluated by analysing the prescriptions.

Results: A total of 400 patients were included in the study. Percentage of indoor patients with one or more AMAs prescribed was $48.75 \%$. The average number of AMAs prescribed was 1.67. Percentage of AMAs prescribed consistent with the National List of Essentials Medicines (NLEM) was $89.73 \%$. The average AMAs duration prescribed was found to be 4.24 days. The most commonly prescribed antibacterial agent in medicine in patients was a beta-lactam AMA with $57.29 \%$ of all prescriptions.

Conclusion: AMR is strongly linked to improper antimicrobial use. This study suggests that certain interventions are required to minimise toxicity and lessen the chances of emergence of resistance. It is therefore proposed that there should be continuous education on rational use of drugs among healthcare professionals.
\end{abstract}

Keywords: Anti-Infective Agents; Drug Resistance, Microbial; Essential Medicines List; Prescription Drug Monitoring Programmes.
(1) Department of Pharmacology, SMS Medical College \& Hospital, Jaipur, India.

Correspondence:

SHIVANKAN KAKKAR

M: +91982827 6839

E: drshivankan@gmail.com

ARTICLE INFO

Received: 22 August 2020

Revision received: 2 September 2020 Accepted: 3 September 2020

\section{Introduction}

Antimicrobial agents (AMAs) have continued to remain the basis of treatment in modern medicine, since their development. ${ }^{1-3}$ AMA use has resulted in a worldwide decline in infection-associated mortality. ${ }^{4,5}$ Today, the most commonly prescribed medication in any hospital settings is an antimicrobial agent. ${ }^{6}$ Judicious use of antimicrobials is rapidly becoming a major public health need. ${ }^{7}$ Inappropriate/improper antimicrobial use can result in increased adverse drug reactions (ADRs), suboptimal therapy, treatment failures, polypharmacy and the most significant of all, the emergence of antimicrobial resistance (AMR). ${ }^{8,9}$

The pattern of antimicrobial use varies in different geographical regions and from centre to centre, depending on the strains of pathogens, the pattern of nosocomial infections, cost and availability of AMA's. In view of the up-and-coming worldwide menace of AMR, there is a requirement to identify patterns of AMA prescribing. Therefore, the present study was designed to

Copyright $\odot 2020$ Jain et al. This is an open access article distributed under the Creative Commons Attribution License (CC BY), which permits unrestricted use, distribution, and reproduction in any medium, provided the original work is properly cited. This article should be cited as follows: Jain M, Kakkar S, Bansal A, Kapadia R. A cross-sectional study of prescribing pattern of antimicrobial agents among inpatients of a tertiary healthcare centre. Scr Med 2020;51(3):203-8. 
study the prescribing pattern of AMAs (according to the World Health Organization's How to investigate AMA use in hospitalised patients: 2012 ${ }^{10}$ among the medicine indoor patients of SMS Medical College \& Hospital, Jaipur, India. This study helped in evaluating, monitoring and suggesting modifications, all in an attempt to rationalise the prescribing practices.

\section{Methods}

The objectives of this observational study were to describe and analyse the prescribing pattern of AMAs among medicine indoor patients of SMS Medical College and Hospital, Jaipur, India and it specifically related to the appropriateness of use of antimicrobials. The administrative permission to conduct the study was obtained from the Medicine Department \& Institutional Research Review Board. The study was a cross-sectional in design. It was conducted for the duration of 12 months (in order to include seasonal variations in diseases). The inclusion and exclusion criteria are listed below.

Inclusion criteria:

1. Patients of any sex, aged 18 to 60 years.

2. Inpatients from the Medicine Department.

3. Patients who gave written informed consent.

Exclusion criteria:

1. ICU admitted patients.

2. Patients on antitubercular or antiretroviral therapy.

3. Post-surgical patients.

Informed consent was obtained in writing from all the patients after fully explaining the study procedure to their satisfaction. This study was approved by the Research committee at the SMS Medical College \& Hospital, Jaipur, India.

Data were collected from the selected Medicine Department inpatients in a predesigned case report form (CRF). The CRF contained dates of admission and discharge, patient demographics, age, sex, outcome, history of presenting illness, general physical examination, diagnosis, investigations, drug details and other relevant information. The data obtained was assessed for the prescribing pattern, as per World Health Organization's How to investigate AMA use in hospitalised patients, $2012 .^{10}$

\section{Statistics}

The sample size at $95 \%$ confidence level expecting $50 \%$ use of antimicrobials (maximum variance) in the Medicine Department of the SMS Hospital. At the precision (relative allowable error) of $10 \%$, minimum of 384 patients were required as a sample size which was further increased and rounded up to 400 patients as a minimum sample size required for the present study (Davey et al, 2005). ${ }^{11}$

Data collected was tabulated and analysed using descriptive statistical tools (mean, standard deviation and percentage wherever applicable). Probability $\mathrm{p}<0.05$ was considered as significant.

\section{Results}

A total of 400 patients from the Medicine Department were included in the study. Out of these patients, 281 (70.25\%) were males while 119 (29.75 $\%$ ) were females. The age of the study patients was in categories; 59 patients (14.75\%) were aged 18-20, 66 patients (16.5\%) were aged 21-30, 87 patients (21.75\%) were aged 31-40 and 188 patients (47\%) were above 40 years. The most commonly prescribed AMAs were antibacterials with a frequency of 285 (85.84\%).

The most commonly prescribed antibacterial group in medicine in patients were beta-lactams with a frequency of 165 (57.29 \%) (Table 1). 0xazolidinones and tetracyclines accounted for 37 $(12.85 \%)$ and $32(11.11 \%)$ respectively. The least prescribed antibacterial was aminoglycosides with a frequency of $1(0.35 \%)$.

Table 1: Frequencies of prescription of antibacterial agents in inpatients of a tertiary healthcare centre

\begin{tabular}{clcc}
\hline $\begin{array}{l}\text { Name of } \\
\text { No. Antibacterial Agent }\end{array}$ & $\begin{array}{l}\text { Number of } \\
\text { patients prescribed }\end{array}$ & $\begin{array}{l}\text { Percentage of } \\
\text { patients prescribed }\end{array}$ \\
\hline 1 Aminoglycosides & 1 & $0.35 \%$ \\
\hline 2 & Beta-lactams & 165 & $57.29 \%$ \\
\hdashline 3 & Lincosamides & 25 & $8.68 \%$ \\
\hline 4 & Macrolides & 9 & $3.13 \%$ \\
\hline 5 & Oxazolidinones & 37 & $12.85 \%$ \\
\hline 6 & Quinolones & 16 & $5.56 \%$ \\
\hline 7 & Tetracyclines & 32 & $11.11 \%$ \\
\hline
\end{tabular}

Conditions recorded on prescription and antimicrobials prescribed are listed in Table 2. 
Table 2: Conditions of inpatient of a tertiary healthcare centre and prescribed antimicrobial agents

\begin{tabular}{|c|c|}
\hline Condition & Antimicrobial agent prescribed \\
\hline Acute pancreatitis & Meropenem (2), Clindamycin (1), Linezolid (1) \\
\hline Alcoholic hepatitis & Ceftriaxone (3), Metronidazole (2) \\
\hline Anaemia & Albendazole (3), Artesunate (1), Cefpirome (1), Ceftriaxone (1), Metronidazole (1) \\
\hline Chronic liver disease & $\begin{array}{l}\text { Linezolid (4), Meropenem (2), Piperacillin (2), Albendazole (1), Ceftriaxone (1), } \\
\text { Clindamycin (1), Metronidazole (1) }\end{array}$ \\
\hline $\begin{array}{l}\text { Chronic obstructive pulmonary } \\
\text { disease (COPD) }\end{array}$ & $\begin{array}{l}\text { Amoxicillin Clavulanate (6), Cefoperazone (3), Ofloxacin (3), Cefpirome (1), } \\
\text { Fluconazole (1) }\end{array}$ \\
\hline $\begin{array}{l}\text { Cerebrovascular incident (CVI), } \\
\text { Stroke/ Paralysis }\end{array}$ & $\begin{array}{l}\text { Ceftriaxone (17), Linezolid (2), Acyclovir (1), Cefpirome (1), Cefuroxime (1), } \\
\text { Metronidazole (1), Moxifloxacin (1), Ticarcillin (1) }\end{array}$ \\
\hline Dengue/ Pyrexia-Thrombocytopenia & Artesunate (2), Ceftriaxone (2), Doxycycline (2), Cefixime (1), Primaquine (1) \\
\hline Diabetes/Complications & Ceftriaxone (4), Moxifloxacin (3), Cefoperazone (1), Doxycycline (1) \\
\hline Encephalopathy & Ceftriaxone (6), Cefoperazone (1) \\
\hline Enteric Fever & Azithromycin (1), Cefixime (1), Ceftriaxone (1) \\
\hline $\begin{array}{l}\text { Guillian-Barre syndrome/ } \\
\text { Muscle weakness }\end{array}$ & Ceftriaxone (4) \\
\hline $\begin{array}{l}\text { Generalised tonic - clonic seizures/ } \\
\text { Seizure disorder }\end{array}$ & Ceftriaxone (6) \\
\hline Hepatitis C & $\mathrm{FDC}(1)$ \\
\hline Infectious diarrhoea & Ceftriaxone (4), Metronidazole (3), Ciprofloxacin (1) \\
\hline Malaria & Artesunate (17), Doxycycline (14), Clindamycin (1), Primaquine (1) \\
\hline Pneumonia & $\begin{array}{l}\text { Ceftriaxone (25), Amoxicillin Clavulanate (9), Cefotaxime (9), Aztreonam (3), } \\
\text { Clindamycin (3), Linezolid (3), Amoxicillin (1), Clarithromycin (1) }\end{array}$ \\
\hline Pyrexia of unknown origin & $\begin{array}{l}\text { Doxycycline (9), Ceftriaxone (8), Artesunate (6), Amoxicillin Clav (5), Azithro- } \\
\text { mycin (4), Levofloxacin (1), Moxifloxacin (1) }\end{array}$ \\
\hline Scrub typhus & Azithromycin (3), Doxycycline (3) \\
\hline Sepsis & $\begin{array}{l}\text { Linezolid (26), Clindamycin (19), Meropenem (16), Aztreonam (7), Artesunate (2), } \\
\text { Piperacillin (2), Cefoperazone (1), Ceftriaxone (1), Moxifloxacin (1) }\end{array}$ \\
\hline Snake Bite/ Unknown Bite & Amoxicillin Clav (2), Linezolid (1), Metronidazole (1), Piperacillin (1) \\
\hline Uper respiratory tract infection & Cefoperazone (1), Doxycycline (1) \\
\hline Urinary tract infection & Levofloxacin (4), Gentamicin (1), Meropenem (1), Norfloxacin (1) \\
\hline
\end{tabular}

Table 3 contains local data for the WHO Prescribing indicators of antimicrobial agents.

\section{Table 3: WHO Prescribing indicators of antimicrobial agents in inpatients of a tertiary healthcare centre}

Percentage of indoor patients with one or more AMAs prescribed
Average number of AMAs prescribed
Percentage of AMAs prescribed consistent with the National List of Essentials Medicines
(NLEM)
Average duration for AMAs prescribed
Pneumonia patients who were prescribed AMAs in accordance with standard treatment
guidelines (STG)
Percentage of AMAs prescribed by their generic names
Additional Indicator: Number of drug sensitivity tests (DST) reported per hospital admis-
sion with AMA prescription

However, the average number of medicines prescribed was found to be 7.12. Proton Pump Inhibitors (PPIs) contributed to being most routinely prescribed among patients.

\section{Discussion}

Antimicrobials used in hospitals for treating infections are often used unacceptably. The study revealed that the percentage of medicine indoor patients with AMAs prescribed was neither very 
high nor extremely low. The percentage of AMAs prescribed consistent with the NLEM suitable. Similarly, the percentage of pneumonia patients who are prescribed AMAs in accordance with standard treatment guidelines (STG) was found to be appropriate. In the study hospital, it was found that the percentage of AMAs prescribed by their generic names is absolute which is most creditable.

The percentage of indoor patients with one or more AMAs prescribed was $48.75 \%$. Although, this is more than the typical values obtained from previous studies from developing countries like Bangladesh (25\%), Malawi (34\%), Tanzania (39 \%) and Indonesia (43.1\%) (Janković et al, 2006 and Massele et al, 2001) ${ }^{12,13}$ but acceptable. It is comparable to the study by Odunsanya et al $(2004)^{14}$ and the study in Iran ${ }^{15}$ that showed the value close to $50 \%$. This study was done for a period of 1 year, so the seasonal variations in diseases were taken into account. Reports from other studies were $60 \%$ (Bosu et al, 2000), ${ }^{16} 60.9$ $\%$ (Otoom et al, 2002), ${ }^{17} 61.9 \%$ (Moghadamnia et al, 2002) ${ }^{18}$ and $72.8 \%$ (Hazra et al, 2000). ${ }^{19}$ Some of the reports from Oman, ${ }^{20}$ Malaysia, ${ }^{21}$ Saudi Arabia $^{22}$ and China ${ }^{23}$ had values of the order of 64 $\%, 67 \%, 72 \%$ and $77.8 \%$ respectively, which are way higher than the one from this study. This indicator finds out the degree of AMA use in hospitals. When used over a period of time, it is able to observe the changing trends.

The average number of AMAs prescribed was 1.67. Indoor patients may receive more than one AMA during the course of their treatment. This prescribing may be warranted or unwarranted. Even duplication occurs in exceptional events. The study from Oman reported the average number to be 2.5, which is higher in comparison to the present study. ${ }^{20}$ In contrast the study from Ghana reported the average number to be 1.1. ${ }^{24}$ Since in the medicine indoor patients who were prescribed AMAs were prescribed an average of 1.67 AMA per hospitalisation, the rate is satisfactory in most situations.

National List of Essentials Medicines (NLEM) represents the medicines that fulfil the priority health care needs of the patients in a hospital, is used to rationalise the use of medicines. Non- adherence to this can have many causes including lack of awareness, not being in conformity and listed medicines (AMAs) not being available at the hospital. Percentage of AMAs prescribed consistent with the NLEM was $89.73 \%$. This indica- tor measures the degree of prescriber adherence to the NLEM. Since a total of $89.73 \%$ of AMAs prescribed were on the NLEM, ${ }^{25}$ this percentage is acceptable, and no further assessment was done to examine adherence. It can be said that the prescribers are aware and in agreement with the list. The routine training programme and workshops in SMS Medical College and Hospital can be attributed to this achievement. These academic activities in a tertiary care teaching hospital give physicians an up to date on treatment protocols. Another factor responsible for this is the fact that the NLEM is also updated regularly and revised accordingly. It can be hoped that in future this indicator would improve even further and there will be a suitable use of AMAs that are on the NLEM.

In SMS Medical College and Hospital the average AMAs duration prescribed was found to be 4.24 days. This indicator measures the strength of patient exposure to AMAs. The number of days on AMAs includes the number of days of all AMAs prescribed for treatment (not prophylaxis). It includes the duration of AMAs prescribed on discharge. Since the average duration of prescribed AMAs was 4.24 days, it conforms to other studies that suggest reducing the duration of antimicrobial use. ${ }^{26}$ Also, this duration of therapy is at par with most international guidelines on antimicrobial use. But the discontinuing the antimicrobials post discharge may have been overlooked.

The adherence with hospital treatment standards is reflected in prescribing AMA of choice as per the STG. ${ }^{27,28}$ Pneumonia patients who were prescribed AMAs in accordance with STG was found to be $81.58 \%$ This indicator measures whether the patient prescription adheres to the treatment guidelines or not. The therapeutic options to pneumonia were analysed according to 2016 guidelines. ${ }^{28}$ In SMS Medical College and Hospital, $81.58 \%$ of pneumonia cases were treated with the recommended AMAs according to the latest guidelines. It suggests that most of the prescribers are aware of these guidelines and are in wilful compliance. Also, all prescribers used the recommended doses as well. This is contrary to the study by Sharma and Kapoor in 2003 which showed that a large number of prescriptions did not match the STG. ${ }^{29}$ However, in the study by Hariharan et al in 2009, 67\% prescriptions adhered to hospital protocol. ${ }^{30}$

In the study hospital, it was found that the percentage of AMAs prescribed by their generic 
names is $100 \%$. This indicator deals with the percentage of AMAs that are prescribed using their generic names, as per the WHO list of international non-proprietary names (INN) (WHO 2008). ${ }^{31}$ The availability of generic drugs in the Government supply and the awareness and agreement of prescribers was looked for. In Rajasthan, after the implementation of free drug scheme, there is strict adherence to generic prescribing in all the Government-run hospitals. In addition, there are Medical Council of India (MCI) guidelines for ensuring the same. ${ }^{32}$ The myth about free drugs being not effective, toxic and contributing to resistance is found to be false in the present study. Since it was ascertained that 100 percent of drugs were prescribed by their generic names, the role of State Government in implementation of MCI guidelines and ensuring compliance of the same is indeed commendable.

The use of AMAs depends on the knowledge of their spectrum. The rate of sensitivity tests done is a measure of the ability of the prescriber to provide rational treatment. The number of drug sensitivity tests reported per hospital admission with AMAs prescribed was found to be $8.21 \%$. This value is lesser in comparison to the Oman study which found that $25 \%$ of drug sensitivity was done. ${ }^{20}$ However, the Chinese study showed that a mere 39 specimens in the 1025 cases were sent for testing. ${ }^{23}$ Whereas, Ider et al (2010) in Mongolia showed values similar to the present study. ${ }^{33}$ This indicator measures the availability of drug sensitivity to establish best possible treatment to infective diseases. Here, since drug sensitivity test was performed for $8.21 \%$ of hospital admissions treated with AMA, an intervention to improve the microbiology lab services may be required to improve this indicator to acceptable limits.

The average number of medicines prescribed was found to be 7.12. However, the study from Pakistan showed polypharmacy of 12 drugs/prescription. ${ }^{34}$ Similarly, polypharmacy was found in GMC, Dhule by Deshmukh et al (2013). ${ }^{35}$ The present study found that among AMAs, the antibacterial agents were prescribed for $85.84 \%$ of patients. Among antibacterials, as the beta-lactams account for $57.29 \%$ of prescriptions; this value corresponds to the worldwide consumption of antimicrobials reported by Boeckel et al (2014) who showed that penicillins and cephalosporins accounted for $37 \%$ and $24 \%$ respectively. ${ }^{36}$ Therefore, the present results correspond to the global trend in antimicrobial consumption.

\section{Conclusion}

Antimicrobial prescribing among medicine in patients at SMS Medical College and Hospital, Jaipur was found to be satisfactory with an area of concern. There are chances of antimicrobial resistance and toxicity of drugs. A practical way to address this urgent need is education and awareness about AMR among prescribers. Rational use of drugs should be promoted, as advocated by the WHO. ${ }^{37}$ Besides, it is recommended to develop a restrictive antimicrobial use policy. This would ensure not only tailored intervention for empirical therapy but adjusted antimicrobial use once drug sensitivity test results are available.

\section{Limitations}

There are several limitations to this study. It is a single centre, observation study describing only the prescribing indicators. A preliminary comparison and evaluation of findings with other hospitals and countries is done. More studies would be required to further monitor, modify and rationalise drug use across the country.

\section{Acknowledgements}

The contributors of the article acknowledge the invaluable inputs and support provided by Departments of Medicine, Microbiology and Community Medicine, SMS Medical College \& Hospital, Jaipur, India.

\section{Conflict of interest}

None.

\section{References}

1. Aminov RI. A brief history of the antibiotic era: lessons learned and challenges for the future. Front Microbiol 2010;1:134.

2. Kilgour FG. Modern Medicine in Historical Perspective. Bull Med Libr Assoc 1962;50(1):42-56.

3. Waksman, S. A. History of the word 'antibiotic.' J Hist Med Allied Sci 1973;28:284-6. 
4. Chambers HF. General principles of antimicrobial therapy. In: Brunton LL, Lazo JS, Parker KL. Goodman \& Gilman's the pharmacological basis of therapeutics. 11th ed. New York: McGeaw-Hill; 2005. p. 1095-1110.

5. Adedeji WA. The Treasure Called Antibiotics. Ann Ib Postgrad Med 2016;14(2):56-7.

6. Meher BR, Mukharjee D, Udayshankar. A study on antibiotic utilization pattern in a general medicine ward of a tertiary care teaching hospital. J Chem Pharm Res 2014;6(7): 1847-49.

7. Remesh A, Salim S, Gayathri AM, Nair U, Retnavally KG. Antibiotics prescribing pattern in the in-patient departments of a tertiary care hospital. Arch Pharm Pract 2013;4:71-6.

8. Kisuule F, Wright S, Barreto J, Zenilman J. Improving antibiotic utilization among hospitalists: a pilot academic detailing project with a public health approach. J Hosp Med 2008;3(1):64-70.

9. Kanish Ravika, Gupta Kanchan, Juneja Shivani, Bains HS, Kaushal Sandeep. Prescribing pattern of antibiotics in the department of pediatrics in a tertiary care medical college hospital in Northern India. Asian J Med Sci 2014;5(4):69-72.

10. Systems for Improved Access to Pharmaceuticals and Services (SIAPS). How to Investigate Antimicrobial Use in Hospitals: Selected Indicators. Published for the U.S. Agency for International Development by the Strengthening Pharmaceutical Systems Program. Arlington, VA: Management Sciences for Health; 2012. Available at http://siapsprogram.org/wp-content/uploads/2012/12/12-096-AMR-Hospital-Indicator-Manual.English.final-11.13.12.pdf. Accessed 15 June 2020.

11. Davey P., Brown E, Fenelon L, Finch R, Gould I, Hartman $G$, et al. Interventions to improve antibiotic prescribing practices for hospital inpatients. Cochrane Database Syst Rev 2005 Oct 19;(4):CD003543. doi: 10.1002/14651858.CD003543.pub2.

12. Janković SM, Vasić LjM, Maksimović MR, Ćupurdija VB, Kostić IR, Kovačević ZN. An analysis of drug use indicators in primary care health facilities operating in the city of Kragujevac, Serbia. Gen Pract On-Line 2006;(16):116. https://www.priory.com/fam/Kosovo.htm.

13. Massele AY, Nsimbi SED, Rimoy G (2001). Prescribing habits in church-owned primary health care facilities in Dar ES Salaam and other Tanzanian Coast regions. East Afr Med J 2001;78(10):510-4.

14. Odunsanya 00. Drug use indicators at secondary health care facility in Lagos, Nigeria. J Commun Med Health Care 2004;16(1):21-4.

15. Safaeian L, Mahdanian AR, Hashemi-Fesharaki M, Salami S, Kebriaee-Zadeh J, Sadeghian GH. General physicians and prescribing pattern in Isfahan, Iran. Oman Med J 2011 May; 26(3):205-6.

16. Bosu WK, Ofori-Adjei D. An audit of prescribing practices in Health Care Facilities of the Wassa west district of Ghana. West Afr J Med 2000;19(4):298-303.

17. Otoom S, Batieha A, Hadidi H, Hasan M, Al-Saudi K. Evaluation of drug use in Jordan, using WHO prescribing indicators. East Mediterr Health J 2002;8(4-5):537-43.

18. Moghadamnia AA, Mirbolooki MR, Aghili MB. General Practitioner prescribing patterns in Babul City Islamic Republic of Iran. East Mediterr Health J 2002;8(45):550-5.

19. Hazra A, Tripathi SK, Alam MS (2000). Prescribing dispensing activities at the health facilities of a non-government organization. Natl Med J India 2000;13(14):17782.

20. Al-Yamani A, Khamis F, Al-Zakwani I, Al-Noomani H, Al-Noomani J, Al-Abri S. Patterns of antimicrobial pre- scribing in a tertiary care hospital in Oman. Oman Med J 2016; 31(1):35-9.

21. Hooi PY, Yong CM, Cheong I. A study of the appropriateness of antibiotic use in the medical wards of a tertiary teaching hospital in Malaysia. Int J Clin Pract 2001 May; 55(4):272-4.

22. Al Shimemeri A, Al Ghadeer H, Memish Z. Antibiotic utilization pattern in a general medical ward of a tertiary medical center in Saudi Arabia. Avicenna J Med 2011 Jul; 1(1):8-11.

23. Arya SC. Antibiotics prescription in hospitalized patients at a Chinese university hospital. J Infect 2004;48:117-8.

24. Bosu WK, Ofori Adjei D. Survey of antibiotic prescribing pattern in government health facilities of the Wassa west district of Ghana. East Afr med J 1997 Mar;74(3):138-42.

25. Essential Drug List 2016. Available from http://rmsc. health.rajasthan. gov.in/content/dam/doitassets/Medical-and-Health-Portal/rajasthan-medical-corporation/ pdf/EDL_Rajasthan.pdf

26. Ferrer R, Artigas A, Suarez D, Palencia E, Levy MM, Arenzana A, Perez XL, Sirvent JM: Effectiveness of treatments for severe sepsis: a prospective, multicenter, observational study. Am J Respir Crit Care Med 2009; 18:861-6.

27. Standard Treatment Guidelines 2012 -RMSC. Available from http://rmsc.health.rajasthan.gov.in/content/dam/ doitassets/Medical-and -Health-Portal/rajasthan-medical-corporation/pdf/Standard $\% 20 \quad$ Treatment $\% 20$ Guidelines/Prelims-2012.indd.pdf

28. National Centre for Disease Control. National Treatment Guidelines for Antimicrobial Use in Infectious Diseases. New Delhi: National Centre for Disease Control, Ministry of Health and Family Welfare; 2016. Available at: http://www.ncdc.gov.in/writereaddata/linkimages/ AMR_guideline7001495889.pdf. Accessed 20 July 2020.

29. Sharma P, Kapoor B. Study of prescribing pattern for rational drug therapy. Jammu Kashmir Sci 2003;5:107-9.

30. Hariharan S, Pillai G, McIntosh D, Bhanji Z, Culmer L, Harper-McIntosh K. Prescribing patterns and utilization of antimicrobial drugs in a tertiary care teaching hospital of a Caribbean developing country. Fundam Clin Pharmacol 2009;23:609-15.

31. WHO Drug Information. 2008. Available from http:// apps.who.int/ medicinedocs/index/assoc/s14883e/ s14883e.pdf. Accessed 20 July 2020.

32. GOI. Gazette Notification. 2016. Available from https:// www. mciindia.org/documents/e_Gazette_Amendments/Ethics-08.10.2016.pdf Acessed 19 July 2020.

33. Ider BE, Clements A, Adams J, Whitby M, Muugolog T. Prevalence of hospital-acquired infections and antibiotic use in two tertiary Mongolian hospitals. J Hosp Infect 2010;75: 214-9.

34. Najmi MH, HafijRA, Khan I, Fazli FR. Prescribing practices: An overview of three teaching hospitals in Pakistan. J Pak Med Assoc1998 Mar;48(3):73-7.

35. Deshmukh VS, Khadke VV, Patil AW, Lohar PS. Study of prescribing pattern of antimicrobial agents in indoor patients of a tertiary care hospital. Int J Basic Clin Pharmacol 2013;2:281-5.

36. Van Boeckel TP, Gandra S, Ashok A, Caudron Q Grenfell BT, Levin SA, et al. Global antibiotic consumption 2000 to 2010: an analysis of national pharmaceutical sales data. Lancet Infect Dis 2014 Aug;14(8):742-50.

37. WHO. Policy perspectives on medicines: Promoting rational use for medicines: core components. 2002. Available from http://apps.who. int/medicinedocs/en/d/ Jh3011e/. Accessed 18 July 2020. 